\title{
Distribution of cathepsin L in human umbilical cord tissues
}

\author{
Tomasz Gogiel ${ }^{\bowtie}$, Małgorzata Wolańska', Zofia Galewska', Piotr Kinalski², \\ Krzysztof Sobolewski' and Lech Romanowicz ${ }^{1}$
}

1Department of Medical Biochemistry, Medical University of Białystok, Poland; 2Department of Gynaecology and Obstetrics, Provincial Hospital in Bialystok, Bialystok, Poland

\begin{abstract}
The extracellular matrix components show specific distribution patterns within various structures of the umbilical cord, among which Wharton's jelly is especially collagenrich tissue. Cathepsin $L$ is a potent cysteine protease engaged in degradation of extracellular matrix proteins, including collagens. We evaluated the activity and expression of cathepsin $L$, and the inhibitory effect of cysteine protease inhibitors in the umbilical cord arteries, vein and Wharton's jelly. Cathepsin L activity and anti-papain inhibitory effect of cysteine protease inhibitors were quantified in extracts of separated umbilical cord tissues using fluorogenic substrates. The results were calculated per DNA content. The enzyme expression was assessed by Western immunoblotting. The active cathepsin $L$ activity (without activation by pepsin digestion), its percentage in the total activity (after pepsin activation), and the expression of the mature single-chain enzyme were the lowest in the umbilical cord arteries and the highest in Wharton's jelly. The effect of cysteine protease inhibitors showed similar distribution as in the case of the active enzyme, being the highest in Wharton's jelly. Distribution of the activity and expression of mature cathepsin $L$ within the umbilical cord probably results from distinctions in the proenzyme activation process. Differences in the action of cysteine protease inhibitors can partly restrict divergences in the enzyme activity that could reflect its expression alone. Differential enzyme action seems to contribute to tissue-specific collagen turnover within the umbilical cord cells, especially those of Wharton's jelly.
\end{abstract}

Key words: cathepsin L, cysteine protease inhibitors, umbilical cord, umbilical cord artery, umbilical cord vein, Wharton's jelly

Received: 26 January, 2017; revised: 06 April, 2017; accepted: 30 April, 2017; available on-line: 09 August, 2017

e-mail: tgogiel@umb.edu.pl

Abbreviations: AMC, 7-amido-4-methylcoumarin; CATB, cathepsin B; CATL, cathepsin L; CHAPS, 3-[(3-cholamidopropyl)dimethylammonio]-1-propane-sulphonate; CPIs, cysteine protease inhibitors; EDTA, ethylenediaminetetraacetic acid; GAPDH, glyceraldehyde-3-phosphate dehydrogenase; ECM, extracellular matrix; GAG; glycosaminoglycan; hc-CATL, heavy chain of double-chain cathepsin L; IGF-I, insulin-like growth factor-I ; IGF BP-3, insulinlike growth factor binding protein-3; pro-CATB, procathepsin B; pro-CATL, procathepsin L; SC-CATL, single-chain cathepsin L; TGF- $\beta$, transforming growth factor-beta; UC, umbilical cord; UCA, umbilical cord artery; UCV, umbilical cord vein; WJ, Wharton's jelly; Z-FRAMC, benzyloxycarbonyl-phenylalanyl-arginyl-7-amido-4-methylcoumarin; Z-FY(tBu)-DMK, Z-Phe-Tyr(t-Bu)-diazomethylketone

\section{INTRODUCTION}

The vascular system of the placenta plays a key part in the intrauterine foetal growth and development (Bole- hovská et al., 2015). The umbilical cord (UC) is the vital life-sustaining structure connecting the foetus with the placenta (Can \& Karahuseyinoglu, 2007; Romanowicz \& Galewska, 2011; Corrao et al., 2013; Bosselmann \& Mielke, 2015). It contains one vein delivering oxygenated and nutrient-rich blood to the foetus and two arteries carrying deoxygenated blood and metabolic waste products to the placenta (Romanowicz \& Galewska, 2011; Bosselmann \& Mielke, 2015). The vascular wall is rich in many extracellular matrix (ECM) macromolecules (collagen, elastin, proteoglycans and glycoproteins) necessary for its proper structure and functions (Ferguson \& Dodson, 2009; Romanowicz \& Galewska, 2011).

The umbilical cord vessels are embedded in Wharton's jelly (WJ), which is a mucous connective tissue consisting of scarce cells immersed in abundant ECM components, mainly collagen, hyaluronate and several proteoglycans with various sulphated glycosaminoglycan (GAG) chains (Sobolewski et al., 1997; Gogiel et al., 2003; Can \& Karahuseyinoglu, 2007; Ferguson \& Dodson, 2009; Corrao et al., 2013). The abundant ECM components in this tissue provide hydration and supramolecular scaffold that protects its vessels from constriction, overdistension, torsion and bending, caused by foetal grasping or movements and uterine contractions (Can \& Karahuseyinoglu, 2007; Ferguson \& Dodson, 2009; Corrao et al., 2013; Bosselmann \& Mielke, 2015). Additionally, the Wharton's jelly is a rich storage of numerous peptide growth factors that support stromal cells proliferation, differentiation, synthesis and remodelling of the ECM (Sobolewski et al., 2005; Corrao et al., 2013).

ECM homeostasis is conditioned on balanced secretion and degradation of its various components (Corrao et al., 2013) and matrix remodelling is highly affected by activities of proteolytic enzymes, such as cysteine cathepsins (Lalmanach et al., 2015). These include the lysosomal endopeptidase cathepsin L (EC 3.4.22.15; CATL), a member of the papain family of cysteine proteinases that is ubiquitously expressed in many cells and tissues (Kirschke, 2004; Turk et al., 2012; Tan et al., 2013; Pišlar \& Kos, 2014; Lalmanach et al., 2015; Sudhan \& Siemann, 2015). It is synthesized as a preproenzyme consisting of 333 amino acid residues, processed to an inactive latent precursor and transported to lysosomes (Ishidoh et al., 1998; Tan et al., 2013). The proenzyme is converted to a mature single-chain CATL (devoid of the propeptide), which is further cleaved into a double-chain form (Ishidoh et al., 1998).

Cathepsin L plays a major role in the lysosomal degradation of both intracellular and endocytosed proteins (Sudhan \& Siemann, 2015) under acidic conditions (Kirschke, 2004; Turk et al., 2012; Tan et al., 2013; Sud- 
han \& Siemann, 2015). The enzyme cleaves a wide range of ECM components, including collagens, proteoglycans, fibronectin, laminin and elastin (Ishidoh \& Kominami, 1995; Kirschke, 2004; Turk et al., 2012; Tan et al., 2013; Sudhan \& Siemann, 2015). Moreover, it can indirectly facilitate matrix breakdown by proteolytic activation of latent proforms of other degrading enzymes, including urokinase plasminogen activator (Kirschke, 2004; Sudhan \& Siemann, 2015), pro-heparanase (Sudhan \& Siemann, 2015), other cathepsins (Turk et al., 2012; Lalmanach et al., 2015; Sudhan \& Siemann, 2015), and certain matrix metalloproteinases (Kirschke, 2004; Turk et al., 2012; Lalmanach et al., 2015; Sudhan \& Siemann, 2015) or by cleavage and inactivation of inhibitors, such as alpha 1 -proteinase inhibitor and secretory leucoprotease inhibitor (Lalmanach et al., 2015).

The activity of CATL is regulated by endogenous cysteine protease inhibitors (CPIs) of the cystatin superfamily including stefins, cystatins and kininogens (Turk et al., 2012; Novinec \& Lenarčič, 2013; Pišlar \& Kos, 2014; Lalmanach et al., 2015; Sudhan \& Siemann, 2015). These competitive, reversible, tight-binding, proteinaceous inhibitors (Turk et al., 2012; Novinec \& Lenarčič, 2013; Pišlar \& Kos, 2014; Lalmanach et al., 2015) are widespread in human cells, tissues and body fluids (Turk et al., 2012; Novinec \& Lenarčič, 2013; Pišlar \& Kos, 2014; Lalmanach et al., 2015), where they trap and neutralize an excessive peptidase activity (Pišlar \& Kos, 2014). A balance between active cysteine cathepsins and CPIs is crucial for proper cellular and tissue homeostasis (Novinec \& Lenarčič, 2013).

The distribution of cathepsin $\mathrm{L}$ and cysteine protease inhibitors in the umbilical cord has not hitherto been investigated. Therefore, we decided to compare the activity and expression of CATL, and the inhibitory effect of CPIs in the umbilical cord arteries, umbilical cord vein and in Wharton's jelly of healthy newborns.

\section{MATERIALS AND METHODS}

The investigation protocol was approved by the Committee for Ethics and Supervision on Human and Animal Research of the Medical University of Białystok, Poland (Approval No R-I-002/169/2011; 28 April, 2011).

Tissue material. Studies were performed on the umbilical cord arteries (UCAs), umbilical cord veins (UCVs) and Wharton's jelly taken from 10 newborns delivered at term by healthy mothers aged 20-30. The tissue samples and extract preparation were the same as we previously described in detail (Gogiel et al., 2012).

Cathepsin L activity assay. Cathepsin L activity was measured fluorometrically as previously described (Kirschke et al., 1982) with modifications including performing the assay in 96-well black microtiter plates. The final incubation mixture $(200 \mu \mathrm{l})$ contained tissue extract with fluorogenic substrate benzyloxycarbonyl-phenylalanyl-arginyl-7-amido-4-methylcoumarin (Z-FR-AMC; Peptide Institute, Inc.; $10 \mu \mathrm{M}$ ) in $0.1 \mathrm{M}$ citrate/phosphate buffer $\mathrm{pH}$ 5.5, supplemented with $3 \mathrm{mM}$ ethylenediaminetetraacetic acid (EDTA), $4 \mathrm{mM}$ dithiothreitol, $0.05 \%$ (w/v) 3-[(3-cholamidopropyl)-dimethylammonio]-1-propane-sulphonate (CHAPS), 7.5 $\mu \mathrm{M}$ pepstatin and the specific cathepsin B inhibitor $\mathrm{N}$-(L-3-trans-propylcarbamoyloxirane-2-carbonyl)-L-isoleucyl-L-proline (CA-074; Bachem; $1 \mu \mathrm{M}$ ), added to avoid interference of cathepsin B (Werle et al., 1995). Fluorescence of the released 7-amido-4-methylcoumarin (AMC) was read continuously at 1-minute intervals with a Tecan Infinite ${ }^{\circledR} 200$ PRO multimode microplate reader (Tecan Group Ltd., Männedorf, Switzerland) at the excitation and emission wavelengths of 354 and 442 $\mathrm{nm}$, respectively. Measurements were standardised with $10 \mu \mathrm{M}$ AMC. Initial rates were monitored for less than $5 \%$ of total substrate conversion. Duplicate samples additionally contained the cathepsin L-specific inhibitor Z-Phe-Tyr(t-Bu)-diazomethylketone (Z-FY(tBu)-DMK, Enzo Life Sciences; $10 \mu \mathrm{M})$. The difference between values for samples without and with $\mathrm{Z}-\mathrm{FY}(\mathrm{tBu})-\mathrm{DMK}$ corresponded to CATL activity. One unit of enzyme activity $(\mathrm{U})$ was defined as the amount of enzyme releasing $1 \mu \mathrm{mol}$ of product (AMC) per minute at $37^{\circ} \mathrm{C}$.

Latent CATL was activated with pepsin as previously described (Gianotti et al., 2008) with modifications. Briefly, $100 \mu \mathrm{l}$ of UCA, UCV and WJ extracts, $100 \mu \mathrm{M}$ AMC in the extraction buffer (standard), or the extraction buffer alone (negative control) were incubated with $100 \mu$ l

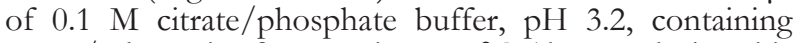
$7 \mathrm{mg} / \mathrm{ml}$ pepsin, for $60 \mathrm{~min}$ at $37^{\circ} \mathrm{C}$ (the $\mathrm{pH}$ during this step was 3.5). Duplicate samples were kept on ice in the absence of pepsin. The reaction was stopped by adding $300 \mu \mathrm{l}$ of $0.1 \mathrm{M}$ citrate/phosphate buffer, $\mathrm{pH}$ 6.1, containing $4 \mathrm{mM}$ EDTA and $25 \mu \mathrm{M}$ pepstatin. All the samples were then subjected to CATL activity assay. The activities measured for samples incubated with pepsin were defined as total CATL activities. The values for samples incubated without pepsin represented active CATL activities. The differences between total and active CATL activities corresponded to the latent enzyme activities.

Determination of inhibitory effect of CPIs in the UC tissue extracts. The inhibitory effect of cysteine proteinase inhibitors was assayed against papain after heat treatment of tissue extracts (at $100^{\circ} \mathrm{C}$ for $10 \mathrm{~min}$ ), using fluorogenic substrate, Z-FR-AMC, as we previously described (Gogiel et al., 2015). One unit of CPI effect (U) was defined as the amount of inhibitory proteins required to totally inhibit the release of $1 \mu$ mol of product (AMC) by papain per minute at $37^{\circ} \mathrm{C}$.

Western blot analysis. Samples of UCA, UCV and WJ extracts were mixed with a cocktail of protease inhibitors (catalogue number P8340; Sigma) at a ratio 100:1 (v/v). They were normalized according to their DNA content $(0.8 \mu \mathrm{g})$ and resolved on $10 \%$ SDS-polyacrylamide gel under reducing conditions according to the method of Laemmli (1970). Separated proteins were transferred to nitrocellulose membranes (Sigma) and probed with rabbit polyclonal antibodies against human cathepsin L (catalogue number 219387; Merck; 1:2000), overnight at $4^{\circ} \mathrm{C}$. Bound antibodies were detected using horseradish peroxidase-conjugated mouse anti-rabbit secondary antibody (clone RG-16, catalogue number A2074; Sigma; 1:20000) and developed with SuperSignal West Pico Chemiluminescent Substrate (Thermo Scientific). The blots were striped and reprobed with anti-glyceraldehyde-3-phosphate dehydrogenase (GAPDH) rabbit polyclonal antibody (catalogue number G9545; Sigma; $1: 20000$ ), overnight at $4^{\circ} \mathrm{C}$, followed by an incubation with alkaline phosphatase-conjugated goat anti-rabbit secondary antibody for 1 hour at room temperature and detection with BCIP/NBT reagent (catalogue number B1911; Sigma).

Determination of DNA content in the UC tissues. DNA concentration was determined by the fluorimetric method of Downs and Wilfinger (1983), with the use of bis-benzimidazole Hoechst 33258 dye (Sigma) and modifications, as we previously described (Gogiel et al., 2015). 


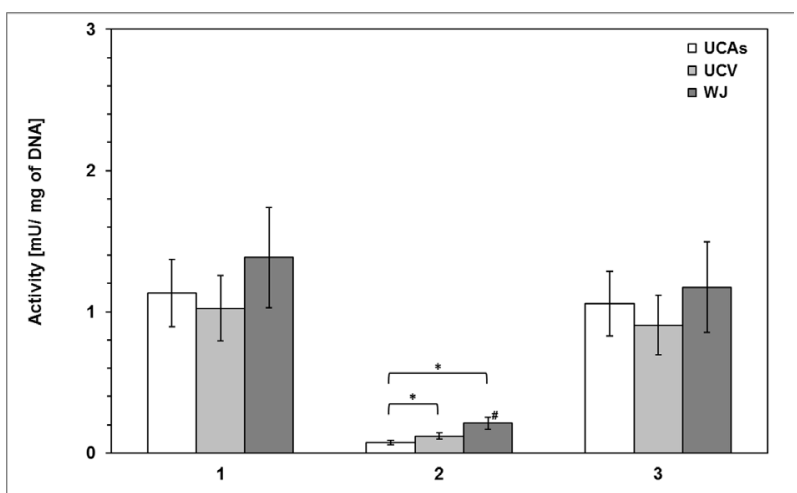

Figure 1. Activity of cathepsin $L$ in umbilical cord tissues. The extracts of umbilical cord arteries (UCAs), umbilical cord vein (UCV) and Wharton's jelly (WJ) were assayed using Z-FR-AMC as a fluorogenic substrate and Z-FY(tBu)-DMK as a specific inhibitor. Mean values \pm SEM $(n=10)$ of total (1), active (2) and latent (pepsin-activatable; 3 ) cathepsin L activities per milligram of DNA contained in the examined tissues are shown. Data were analyzed using Friedman repeated-measures ANOVA $(\# P<0.05)$ and post-hoc Wilcoxon signed-ranks testing corrected for multiple comparisons by the Bonferroni-Holm method $\left({ }^{*} P<0.05\right)$.

Statistical analysis. Data are shown as the mean values from 10 assays and standard errors of mean (SEM). Friedman ANOVA was performed followed by multiple post-hoc Wilcoxon signed-ranks tests corrected by Bonferroni-Holm method. Differences in results with $P<0.05$ were assumed as statistically significant. Statistical analysis was carried out using Statistica 10 software (StatSoft, Inc; Hill \& Lewicki, 2007).

\section{RESULTS}

\section{Cathepsin L activity}

The activities of cathepsin $\mathrm{L}$ calculated per milligram of DNA in UC tissues are presented in Fig. 1. Both total and latent CATL activities were almost evenly distributed within the UC tissues (Fig. 1, parts 1 and 3, respectively;

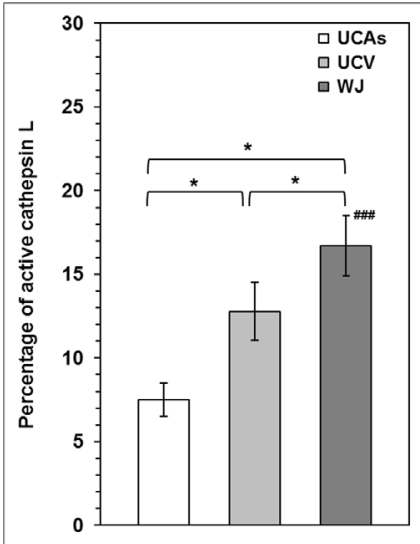

Figure 2. Active cathepsin $\mathrm{L}$ as the percentage of the total enzyme activity in umbilical cord tissues.

Cathepsin $L$ activities in umbilical cord arteries (UCAs), umbilical cord vein (UCV) and Wharton's jelly (WJ) were determined using Z-FR-AMC as a substrate and Z-FY(tBu)-DMK as a specific inhibitor. Active and total enzyme activities were measured in individual tissue extracts. Mean values \pm SEM $(n=10)$ are presented. Data were analyzed with Friedman ANOVA (\#\#\#, $P<0.001)$ followed by post-hoc Wilcoxon signed-ranks tests with Bonferroni-Holm corrections $\left({ }^{*} P<0.05\right)$. all $P>0.05$ ). On the other hand, the active CATL activity in UCV was over 1.5 times higher, and that of WJ - nearly 3 times higher than in the UCAs (Fig. 1, part 2; both $P<0.05$ ). The values for active CATL (Fig. 1, part 2) were much lower than those of the total and latent enzyme (Fig. 1, parts 1 and 3, respectively).

\section{Active cathepsin L activity as the percentage of the total enzyme activity}

In order to evaluate the efficiency of CATL activation we decided to compare the activities of the active enzyme expressed as percentages of those of the total enzyme, measured for individual materials. It is apparent from Fig. 2 that the activity of active CATL constituted only $7.5-16.7 \%$ of the total activity, depending on the UC tissue (Fig. 2). As can be seen, this percentage for the WJ was over 2 times higher than for the UCAs and almost 1.5 times higher than for the UCV, and that of UCV - over 1.5 times higher when compared with the UCAs (Fig. 2, all $P<0.05$ ).

\section{Inhibitory effect of cysteine protease inhibitors}

Figure 3 shows CPI effect of the UC tissue extracts. This effect in the WJ was over 4.5 times higher than in the case of UCAs and about 3 times higher than in the $\mathrm{UCV}$, whereas that of UCV - over 1.5 times higher than in the UCAs (Fig. 3, all $P<0.05$ ).

\section{Expression of cathepsin $\mathrm{L}$ protein in the umbilical cord tissues}

Western immunoblot analysis of cathepsin L protein expression in the extracts of UC tissues is presented in Fig. 4. Several specific bands were detected. These included: bands of $37-41 \mathrm{kDa}$, corresponding to procathepsin L (pro-CATL; Kirschke, 2004), 34 kDa band, probably reflecting the presence of partial zymogen activation intermediate (see asterisk; Reilly et al., 1989), $28 \mathrm{kDa}$ band of the single-chain mature CATL (sc-CATL), and $24 \mathrm{kDa}$ band of a heavy chain of the fully mature double-chain enzyme (hc-CATL; Kirschke, 2004). The intensity of pro-CATL bands (37-41 kDa)

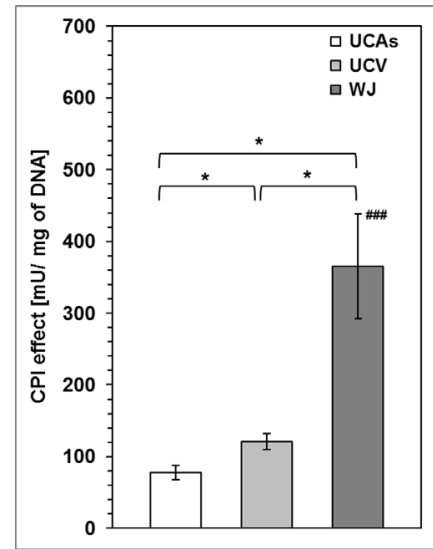

Figure 3. Inhibitory effect of cysteine proteinase inhibitors (CPI) from umbilical cord tissues.

The CPI effect of heat-treated extracts of umbilical cord arteries (UCAs), umbilical cord vein (UCV) and Wharton's jelly (WJ) against active site titrated papain was measured using Z-FR-AMC as a substrate. Mean values \pm SEM $(n=10)$, expressed per milligram of DNA contained in umbilical cord tissues, are presented. Data were analyzed with Friedman ANOVA (\#\#\#, $P<0.001)$ followed by posthoc Wilcoxon signed-ranks tests with Bonferroni-Holm corrections $\left({ }^{*} P<0.05\right)$. 


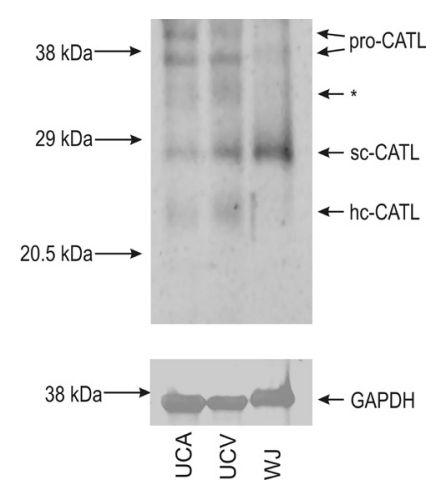

Figure 4. Western immunoblot analysis of cathepsin $L$ in pooled extracts of umbilical cord tissues.

Samples were applied based on DNA content $(0.8 \mu \mathrm{g} /$ lane). Proteins were resolved by SDS-PAGE (10\%) under reducing conditions and immunoblotted with antibodies to human cathepsin L (upper panel) and human GAPDH (lower panel). UCA - umbilical cord artery; UCV - umbilical cord vein; WJ - Wharton's jelly. The positions of pre-stained molecular mass markers (catalogue number 1610318; Bio-Rad Laboratories) which were pre-calibrated with unstained standards (catalogue number 17-0446-01; Pharmacia) by SDS/PAGE are indicated on the left. Immunolabelled cathepsin $\mathrm{L}$ bands are marked on the right: pro-CATL - procathepsin L; scCATL - single-chain mature cathepsin L; hc-CATL - heavy chain of fully processed double-chain mature cathepsin L; an intermediate form of partially processed pro-CATL is marked with asterisk.

was highest in the UCAs (Fig. 4, lane 1), slightly lower for the UCV (lane 2) and very weak for WJ (lane 3). The intermediate band of $34 \mathrm{kDa}$ was more intense for the UCV (lane 2) than for the UCAs (lane 1), but only trace for WJ (lane 3). In contrast, the band of sc-CATL $(28 \mathrm{kDa})$ was stronger for the UCV (lane 2) than for the UCAs (lane 1) and by far the most prominent for WJ (lane 3). On the other hand, the intensity distribution of hc-CATL band $(24 \mathrm{kDa})$ was similar to that of the intermediate band, being more prominent for the UCV (lane 2) than for the UCAs (lane 1), and only trace for WJ (lane 3). However, the overall intensity of the two mature CATL bands was highest for WJ because of that of the single-chain form, which was the most distinct in the whole blot (Fig. 4, lanes 1-3).

\section{DISCUSSION}

Cathepsin L plays major biological role in various physiological processes. Apart from being involved in general protein turnover, it participates in antigen presentation, cardiac function, skin homeostasis, bone resorption, reproduction, cell development and differentiation (Kirschke, 2004; Turk et al., 2012; Novinec \& Lenarčič, 2013; Tan et al., 2013; Lalmanach et al., 2015; Sudhan \& Siemann, 2015). It was suggested to be necessary for normal embryo implantation and development, uterine decidualization and placentation (Afonso et al., 1997). The umbilical cord is a foetus-derived structure; therefore it may provide unique insight into processes taking place within the developing foetus (Ferguson \& Dodson, 2009; Corrao et al., 2013).

We investigated the distribution of cathepsin $\mathrm{L}$ in all the umbilical cord tissues. The catalytically active CATL is located within the cells (Tan et al., 2013; Sudhan \& Siemann, 2015), therefore we quantified its activity against DNA content, which is widely used to assess the cell number in tissues. The results presented here showed that active (not pepsin-treated) CATL activity, calculated per milligram of DNA, was highest in WJ and lowest in the UCAs. These differ- ences strongly correlated with those of the expression of mature CATL forms, as assessed by Western immunoblotting.

It is known that the activity of lysosomal cysteine proteases is a delicate balance of their synthesis, targeting, zymogen activation, inhibition by endogenous inhibitors and degradation (Turk et al., 2012). It should be noted that the total CATL activity was similar in both $\mathrm{UC}$ vessels and in WJ, thus pointing to comparable biosynthetic activity of cells from all UC tissues. Therefore, the observed differences in the activity and expression of the mature enzyme seem to be mainly caused by divergences in the process of pro-cathepsin $\mathrm{L}$ activation. It is supported by a positive correlation of active CATL activity and its percentage in the total enzyme activity, as well as by a negative correlation between the expressions of mature enzyme forms and pro-CATL in the UC tissues. These differences may result from specific features of cells residing in the individual tissues of the umbilical cord. The WJ cells, initially described as myofibroblasts (Can \& Karahuseyinoglu, 2007; Ferguson \& Dodson, 2009; Corrao et al., 2013), have properties of mesenchymal stem cells, because they can differentiate towards various mature cell types derived from all the three germ layers (Can \& Karahuseyinoglu, 2007; Corrao et al., 2013). On the other hand, endothelial cells and smooth muscle cells are located in the UC vessels (Can \& Karahuseyinoglu, 2007; Ferguson \& Dodson, 2009). However, mesenchymal stem cells, able to differentiate into (at least) adipocytes and osteoblasts, were reported to reside also within the UCV endothelial/subendothelial layer (Romanov et al., 2003). Such localisation might thus explain an intermediate activation level of pro-CATL in this tissue.

Differences in glycosaminoglycan content and composition can also contribute to dissimilar pro-CATL activation among the UC tissues. It was reported that GAGs can stimulate autocatalytic conversion of pro-CATL into the mature enzyme, with hyaluronate and keratan sulphate being among the most effective (Ishidoh \& Kominami, 1995). It is of interest that both the total GAG content and the amount of hyaluronate and keratan sulphate are much higher in Wharton's jelly in comparison to the UCA wall (Sobolewski et al., 1997). Moreover, the amount of these and the other (heparin, heparan sulphate) strongly activating GAGs tends to be higher in the UCV than in the UCAs, although the total GAG content is similar in all UC vessels (Romanowicz et al., 1998). Therefore, it cannot be excluded that tissue-specific GAG-mediated activation mechanism of pro-CATL mentioned above is responsible for higher expression and activity of mature CATL forms in the UCV, and especially in Wharton's jelly.

The distribution of active CATL activities positively correlated with the amount of collagen in the UC tissues. It was previously reported that Wharton's jelly contains about 4 times more (Sobolewski et al., 1997) and UCV - 3 times more collagen (Romanowicz \& Sobolewski, 2000) in comparison to the UCA wall (Sobolewski et al., 1997), when calculated per gram of defatted tissue. One can estimate that differences in collagen content per cell within UC tissues are much higher, as the amount of DNA negatively correlates with that of CATL, being 4.5 times less abundant in WJ in comparison to the UCAs and almost 2.5 times less than in the UCV, as we reported previously (Gogiel et al., 2012). One may suppose that such differences would be even higher without differential action of CATL, which can moderate an excessive deposition of collagen and other ECM proteins by cells 
of those tissues. Alternatively, the divergent abundance of mature CATL may contribute to prominent collagen accumulation in WJ and, to a lower extent, in the UCV, by indirectly stimulating the ECM production by cells of those tissues. It was reported that CATL is involved in intracellular degradation of insulin-like growth factor binding protein-3 (IGF BP-3; Zwad et al., 2002), which plays the most important role in insulin-like growth factor-I (IGF-I) binding in the umbilical cord (Bańkowski et al., 2002). One may speculate that differences in actual CATL activity distribution in cells of UC tissues contribute to opposite expression pattern of IGF BP-3 as a result of distinctions in its CATL-mediated breakdown. The degradation of this binding protein is thought to increase bioavailability of IGF-I, thus facilitating its binding to cell receptor and leading to enhanced synthesis of ECM components, including collagens (Bańkowski et al., 2002), which are especially abundant in Wharton's jelly (Sobolewski et al., 1997; Ferguson \& Dodson, 2009; Corrao et al., 2013; Bosselmann \& Mielke, 2015) and, to some extent, in the UCV (Romanowicz \& Sobolewski, 2000).

We also determined the total inhibitory effect of CPIs, an important factor controlling the activity of CATL (Turk et al., 2012; Lalmanach et al., 2015; Sudhan \& Siemann, 2015). It is apparent that the combined effect of these inhibitors was lowest in the UCAs and the highest in WJ. It was reported that the expression and secretion of cystatin C, one of the most abundant CPIs (Novinec \& Lenarčič, 2013; Qin et al., 2013), by various cell types, including fibroblasts, can be stimulated by transforming growth factor- $\beta$ (TGF- $\beta$; Sokol \& Schiemann, 2004; Kotajima et al., 2010; Kasabova et al., 2014; Lalmanach et al., 2015). It is of interest that the amount of this factor (per microgram of DNA) is 42-fold higher in WJ than in the UCA (Sobolewski et al., 2005). Therefore, exceptionally high CPI effect in WJ may be caused by the accumulation of TGF- $\beta$ in this tissue.

Differences in the inhibitory effect of CPIs on ECM-degrading cysteine cathepsins in the UC tissues can favour a prominent ECM accumulation in WJ and, to a lower extent, in the UCV, when compared with the UCAs. A positive correlation between CPI effect and mature CATL expression in the examined tissues may contribute to the lower differences in the enzyme action that could be estimated from its expression alone.

It is apparent from our study that the CPI-mature CATL complexes, apart from the proenzyme, can be responsible for the latent enzyme activity (measured after pepsin digestion). Such complexes seem to significantly contribute to a prominent latency of CATL, as the zymogen expression did not significantly prevail over that of the mature enzyme (sc-CATL and hc-CATL) regardless the tissue examined. Moreover, the latent enzyme activity did not correlate with pro-CATL expression. It is especially evident in the case of WJ, which contained almost exclusively single-chain form of CATL, despite similar latent activity of the enzyme compared to the other UC tissues. Thus, the major part of mature enzyme activity can be masked by such inhibitors and can only be detected after their degradation by pepsin. Interactions with intracellular inhibitors of CATL, stefins $\mathrm{A}$ and $\mathrm{B}$, were suggested to completely prevent the detection of the mature enzyme in liver homogenates unless they were preincubated at acidic $\mathrm{pH}$ (Mason et al., 1985). Moreover, substantial amount of CATL in human lung tissue was found to be complexed with kininogen, which resulted in low CATL activity values in the tissue extracts (Werle et al., 1995).
The activity and expression patterns of CATL in the examined tissues differed from those for catepsin B (CATB; EC 3.4.22.1), a ubiquitous lysosomal cysteine proteinase (Aggarwal \& Sloane, 2014). We previously reported that the highest CATB activity and expression are found in Wharton's jelly and such distribution pattern is mainly caused by differences in the expression and the activity of the latent (pepsin-activatable) enzyme (Gogiel et al., 2012). It is apparent that the activity and expression of the two enzymes are regulated independently.

The expression of CATB is augmented by the action of collagen (Koblinski et al., 2002; Roshy et al., 2003) and TGF- $\beta$ (Reisenauer et al., 2007) in various cell types, including fibroblasts. In contrast to that, the expression and activity of CATL are only weakly stimulated by TGF- $\beta$ (Reisenauer et al., 2007). Taking into account the exceptional abundance of collagen (Sobolewski et al., 1997) and TGF- $\beta$ (Sobolewski et al., 2005) in Wharton's jelly, these factors may be responsible for selective prevalence of only CATB (but not CATL) in this tissue.

Individual forms of these two enzymes also show separate distribution patterns within the umbilical cord, probably because of the differences in lysosomal sorting, conversion and activation. Proforms of both CATB (Aggarwal \& Sloane, 2014) and CATL (Ishidoh et al., 1998) undergo conversion to single-chain forms in lysosomes. However, these two proteinases do not co-localize to the same vesicle, pointing to lysosomal sorting pathways typical for each of them (Gopal et al., 2006). Moreover, the proforms of cysteine cathepsins can be constitutively secreted by some cell types, such as macrophages and fibroblasts (Lalmanach et al., 2015). The secretion of procatepsin B (pro-CATB) can be stimulated by collagen (Koblinski et al., 2002), whereas no such effect on pro-CATL secretion was reported. One may suppose that secretion of pro-CATB within the UC tissues positively correlates with the amount of collagen (Sobolewski et al., 1997; Romanowicz \& Sobolewski, 2000). As a result, the cells of WJ and, to a lower extent, those of the UCV, secrete relatively more and retain less proform of CATB for intralysosomal activation than in the case of pro-CATL. One cannot exclude that these processes are responsible for different pattern of the activity and expression of mature CATL, and of pro-CATB, within the UC tissues.

In summary, the results of our present study show that the active CATL activity and the expression of the mature single-chain enzyme are lowest in the UCAs and highest in WJ, which may result from specific features of the proenzyme activation process within the cells of UC tissues. The distribution of CPIs is similar to that of scCATL, therefore it can partly restrict differences in the enzyme activity that could be estimated from its expression alone. Proteolytic action of CATL can favour degradation of collagen and other ECM proteins by cells within UC tissues or, alternatively, may indirectly stimulate the ECM production by increasing bioavailability of IGF-I following IGF BP-3 cleavage. Therefore, differential enzyme effect may contribute to cell-specific collagen turnover within the umbilical cord, especially in Wharton's jelly. Different morphological and biochemical alterations in the umbilical cord tissues were observed in a variety of pregnancy-associated pathological conditions, including genetic disorders, pre-eclampsia, gestational diabetes, foetal distress and growth retardation, and even in foetal death (Ferguson \& Dodson, 2009; Romanowicz \& Galewska, 2011; Corrao et al., 2013). Although our work concerns healthy subjects, it may facilitate future studies explaining several aspects of various prenatal pathological conditions. 


\section{Conflicts of interests}

The authors declare no conflicts of interest.

\section{Acknowledgements}

The study was supported by the Medical University of Białystok, Project No. 113-15659L.

\section{REFERENCES}

Afonso S, Romagnano L, Babiarz B (1997) The expression and function of cystatin $C$ and cathepsin B and cathepsin $\mathrm{L}$ during mouse embryo implantation and placentation. Development 124: 3415-3425

Aggarwal N, Sloane BF (2014) Cathepsin B: multiple roles in cancer. Proteomics Clin Atpl 8: 427-437. doi: 10.1002/prca.201300105

Bańkowski E, Pałka J, Jaworski S (2002) An expression of IGF-binding proteins in normal and pre-eclamptic human umbilical cord serum and tissues. Mol Cell Biochem 237: 111-117

Bolehovská P, Sehnal B, Driák D, Halaška M, Magner M, Novotný J, Švandová I (2015) Changes in placental angiogenesis and their correlation with foetal intrauterine restriction. Ceska Gynekol 80: 144-150

Bosselmann S, Mielke G (2015) Sonographic Assessment of the Umbilical Cord. Geburtshilfe Frauenheilkd 75: 808-818

Can A, Karahusevinoglu S (2007) Concise review: human umbilical cord stroma with regard to the source of fetus-derived stem cells. Stem Cells 25: 2886-2895

Corrao S, La Rocca G, Lo Iacono M, Corsello T, Farina F, Anzalone R (2013) Umbilical cord revisited: from Wharton's jelly myofibroblasts to mesenchymal stem cells. Histol Histopathol. 28: 1235-1244. doi: $10.14670 / \mathrm{HH}-28.1235$

Downs TR, Wilfinger WW (1983) Fluorometric quantification of DNA in cells and tissue. Anal Biochem 131: 538-547

Ferguson VL, Dodson RB (2009) Bioengineering aspects of the umbilical cord. Eur J Obstet Gynecol Reprod Biol 144 (Suppl 1): S108-S113. doi: 10.1016/j.ejogrb.2009.02.024

Gianotti A, Sommer CA, Carmona AK, Henrique-Silva F (2008) Inhibitory effect of the sugarcane cystatin CaneCPI-4 on cathepsins B and $\mathrm{L}$ and human breast cancer cell invasion. Biol Chem 389: 447453. doi: $10.1515 /$ BC. 2008.035

Gogiel T, Bańkowski E, Jaworski S (2003) Proteoglycans of Wharton's jelly. Int J Biochem Cell Biol 35: 1461-1469

Gogiel T, Galewska Z, Romanowicz L (2012) Differential distribution of cathepsin B in human umbilical cord tissues. Acta Biochim Pol 59: 679-684

Gogiel T, Wolańska M, Galewska Z, Kinalski M, Sobolewski K, Romanowicz L (2015) Cathepsin B in human myometrium and in uterine leiomyomas at various stages of tumour growth. Eur J Obstet Gynecol Reprod Biol 185: 140-144. doi: 10.1016/j.ejogrb.2014.12.020

Gopal P, Rehman RU, Chadha KS, Qiu M, Colella R (2006) Matrigel influences morphology and cathepsin B distribution of prostate cancer PC3 cells. Oncol Rep 16: 313-320

Hill T, Lewicki P (2007) STATISTICS: Methods and applications. StatSoft, Tulsa

Ishidoh K, Kominami E (1995) Procathepsin L degrades extracellular matrix proteins in the presence of glycosaminoglycans in vitro. Biochem Biophys Res Commun 217: 624-631

Ishidoh K, Saido TC, Kawashima S, Hirose M, Watanabe S, Sato N, Kominami E (1998) Multiple processing of procathepsin L to cathepsin L in vivo. Biochem Biophys Res Commun 252: 202-207

Kasabova M, Joulin-Giet A, Lecaille F, Gilmore BF, Marchand-Adam

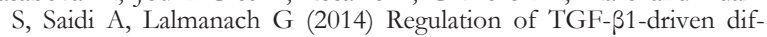
ferentiation of human lung fibroblasts: emerging roles of cathepsin B and cystatin C. I Biol Chem 289: 16239-16251. doi: 10.1074/jbc. M113.542407

Kirschke H (2004) Cathepsin L. In Handbook of Proteolytic Ensymes, 2nd edn, Barrett AJ, Rawlings ND, Woessner JF eds, vol 2, pp $1097-$ 1102. Elsevier Academic Press, London

Kirschke H, Kembhavi AA, Bohley P, Barrett AJ (1982) Action of rat liver cathepsin L on collagen and other substrates. Biochem I 201: $367-372$
Koblinski JE, Dosescu J, Sameni M, Moin K, Clark K, Sloane BF (2002) Interaction of human breast fibroblasts with collagen I increases secretion of procathepsin B. J Biol Chem 277: 32220-32227

Kotajima N, Yanagawa Y, Aoki T, Tsunekawa K, Morimura T, Ogiwara T, Nara M, Murakami M (2010) Influence of thyroid hormones and transforming growth factor- $\beta 1$ on cystatin $C$ concentrations. I Int Med Res 38: 1365-1373

Laemmli UK (1970) Cleavage of structural proteins during the assembly of the head of bacteriophage T4. Nature 227: 680-685

Lalmanach G, Saidi A, Marchand-Adam S, Lecaille F, Kasabova M (2015) Cysteine cathepsins and cystatins: from ancillary tasks to prominent status in lung diseases. Biol Chem 396: 111-130. doi: 10.1515/hsz-2014-0210

Mason RW, Green GD, Barrett AJ (1985) Human liver cathepsin L. Biochem J 226: 233-241

Novinec M, Lenarčič B (2013) Papain-like peptidases: structure, function, and evolution. Biomol Concepts 4: 287-308. doi: 10.1515/bmc2012-0054

Pišlar A, Kos J (2014) Cysteine cathepsins in neurological disorders. Mol Neurobiol 49: 1017-1030. doi: 10.1007/s12035-013-8576-6

Qin Y, Cao X, Yang Y, Shi GP (2013) Cysteine protease cathepsins and matrix metalloproteinases in the development of abdominal aortic aneurysms. Future Cardiol 9: 89-103. doi: 10.2217/fca.12.71

Reilly JJ Jr, Mason RW, Chen P, Joseph LJ, Sukhatme VP, Yee R, Chapman HA Jr (1989) Synthesis and processing of cathepsin L, an elastase, by human alveolar macrophages. Biochem J 257: 493-498

Reisenauer A, Eickelberg O, Wille A, Heimburg A, Reinhold A, Sloane BF, Welte T, Bühling F (2007) Increased carcinogenic potential of myeloid tumor cells induced by aberrant TGF- $\beta 1$-signaling and upregulation of cathepsin B. Biol Chem 388: 639-650

Romanov YA, Svintsitskaya VA, Smirnov VN (2003) Searching for alternative sources of postnatal human mesenchymal stem cells: candidate MSC-like cells from umbilical cord. Stem Cells 21: 105-110

Romanowicz L, Bańkowski E, Jaworski S (1998) Electrophoretic and chromatographic patterns of glycosaminoglycans of the umbilical cord vessels and their alteration in EPH-gestosis. Acta Biochim Pol 45: 805-809

Romanowicz L, Galewska Z (2011) Extracellular matrix remodeling of the umbilical cord in pre-eclampsia as a risk factor for fetal hypertension. J Pregnancy 2011: 542695. doi: 10.1155/2011/542695

Romanowicz L, Sobolewski K (2000) Extracellular matrix components of the wall of umbilical cord vein and their alterations in pre-eclampsia. I Perinat Med 28: 140-146

Roshy S, Sloane BF, Moin K (2003) Pericellular cathepsin B and malignant progression. Cancer Metastasis Rev 22: 271-286

Sobolewski K, Bańkowski E, Chyczewski L, Jaworski S (1997) Collagen and glycosaminoglycans of Wharton's jelly. Biol Neonate 71: 11-21

Sobolewski K, Małkowski A, Bańkowski E, Jaworski S (2005) Wharton's jelly as a reservoir of peptide growth factors. Placenta 26: 747752

Sokol JP, Schiemann WP (2004) Cystatin C antagonizes transforming growth factor beta signaling in normal and cancer cells. Mol Cancer Res 2: 183-195

Sudhan DR, Siemann DW (2015) Cathepsin L targeting in cancer treatment. Pharmacol Ther 155: 105-116. doi: 10.1016/j. pharmthera.2015.08.007

Tan GJ, Peng ZK, Lu JP, Tang FQ (2013) Cathepsins mediate tumor metastasis. World J Biol Chem 4: 91-101. doi: 10.4331/wjbc.v4.i4.91

Turk V, Stoka V, Vasiljeva O, Renko M, Sun T, Turk B, Turk D (2012) Cysteine cathepsins: from structure, function and regulation to new frontiers. Biochim Biophys Acta 1824: 68-88. doi: 10.1016/j. bbapap.2011.10.002

Werle B, Ebert W, Klein W, Spiess E (1995) Assessment of cathep$\sin \mathrm{L}$ activity by use of the inhibitor CA-074 compared to cathepsin B activity in human lung tumor tissue. Biol Chem Hoppe-Seyler 376: $157-164$

Zwad O, Kübler B, Roth W, Scharf JG, Saftig P, Peters C, Braulke $\mathrm{T}$ (2002) Decreased intracellular degradation of insulin-like growth factor binding protein-3 in cathepsin L-deficient fibroblasts. FEBS Lett 510: 211-215 